\title{
Tumor-Stroma Co-Cultures Modify the Invasive Properties of Human Pancreatic Adenocarcinoma Cells Through Different Patterns of Cytokine Secretion and Depending on the Type of Cell Lines.
}

\section{Elena Prieto-García}

Instituto de Investigación Hospital 12 de Octubre: Instituto de Investigacion Hospital 12 de Octubre

C. Vanesa Díaz-García

Instituto de Investigación Hospital 12 de Octubre: Instituto de Investigacion Hospital 12 de Octubre

Alba Agudo-López

Instituto de Investigación Hospital 12 de Octubre: Instituto de Investigacion Hospital 12 de Octubre

Virginia Pardo-Marqués

Instituto de Investigación Hospital 12 de Octubre: Instituto de Investigacion Hospital 12 de Octubre Inés García-Consuegra

Instituto de Investigación Hospital 12 de Octubre: Instituto de Investigacion Hospital 12 de Octubre

Sara Asensio-Peña

Instituto de Investigación Hospital 12 de Octubre: Instituto de Investigacion Hospital 12 de Octubre

Marina Alonso-Riaño

Hospital Universitario 12 de Octubre

\section{Carlos Pérez}

Instituto de Investigación Hospital 12 de Octubre: Instituto de Investigacion Hospital 12 de Octubre

\section{Carlos Gómez}

Hospital Universitario 12 de Octubre

Jorge Adeva

Hospital Universitario 12 de Octubre

\section{Luis Paz-Ares}

Hospital Universitario 12 de Octubre

José A. López-Martín

Hospital Universitario 12 de Octubre

María Teresa Agulló-Ortuño ( $\square$ agullo@h12o.es)

Instituto de Investigación Hospital 12 de Octubre: Instituto de Investigacion Hospital 12 de Octubre https://orcid.org/0000-0002-7617-6681 
Keywords: Pancreatic ductal adenocarcinoma, cancer-associated-fibroblasts, co-cultures, metastasis, epithelial-mesenchymal transition, cytokines, extracellular vesicles.

Posted Date: January 25th, 2021

DOI: https://doi.org/10.21203/rs.3.rs-152450/v1

License: (c) (1) This work is licensed under a Creative Commons Attribution 4.0 International License. Read Full License 


\section{Abstract}

Background: Pancreatic ductal adenocarcinoma (PDAC) is among the most lethal cancer worldwide. One key feature of PDAC is a dense desmoplastic reaction that has become recognized to play important roles in tumor growth, metastasis, and therapeutic resistance. We aim to study tumor-stromal interactions in an in vitro co-culture model between human PDAC cells and fibroblasts.

Methods: Capan-1 or PL-45 human pancreatic tumor cell lines and LC5 fibroblasts were grown in direct or indirect co-cultures, and their relationship regarding migratory and invasive properties were studied by wound-healing and transwell migration assays. Confocal immunofluorescence, ELISA, and western blotting were used to evaluate the expression of activation markers. Cytokines arrays were performed to identify secretome profiles associated to migratory and invasive properties of tumor cells. Extracellular vesicles production was examined by ELISA and transmission electron microscopy.

Results: Co-cultures conditions increased FGF-7 secretion and a-SMA expression, characterizing fibroblasts activation; and decreased epithelial marker E-cadherin in tumor cells, suggesting that they suffer epithelial-to-mesenchymal transition. The expression pattern of cytokine secretion differed under co-culture conditions versus monocultures, with GM-CSF, GRO a/b/g, GRO-a, IL-6, IL-8, MCP-1, and RANTES being the most affected. A greater number of exosomes with higher amount of proteins was quantified in co-cultures assays. Interestingly, tumor cells and fibroblasts migrate as a package, with tumor cells in the middle surrounded by fibroblasts, and maximizing the contact between cell to cell.

Conclusions: We show different migratory properties in tumor and stromal cells, and we draw a different mechanism for tumor spread through a cooperative migration between tumor cells and activated fibroblasts. Furthermore, we found that the level of IL-6 mainly, in addition to other cytokines, change significantly in co-cultures conditions with respect to monocultures, and this could affect the invasive and migratory capacities of cells. Targeting the interaction between tumor cells and tumor microenvironment might represent a novel therapeutic approach to advance pancreatic carcinoma.

\section{Background}

Pancreatic ductal adenocarcinoma (PDAC) is among the most lethal tumors worldwide, with a 5-year overall survival around 9\% (1). Unfortunately, many chemotherapeutic agents, radiation and targeted therapies have failed to improve survival. This lack of success has been mainly associated with late diagnosis and chemoresistance of the disease $(2,3)$. The increasing knowledge of cellular and molecular mechanisms involved in pancreatic carcinogenesis has also failed to translate into new therapeutic approaches. This may be due in part, to the fact that most studies focus mainly on targeting tumor cells and obviate the complex surrounding microenvironment, which plays an important role in the evolution of the disease $(4,5)$.

One of the hallmarks of PDAC is the formation of an abundant and dense stroma wrapping tumor cells, termed desmoplastic reaction, which leads to an increase in the interstitial pressure, and hence, to poor 
tissue perfusion and hypoxia. This tumor-associated stroma may represent up to $80 \%$ of the tumor mass in the majority of patients (6). Although initially it was thought that this component was a barrier limiting tumor progression, today its pro-tumor properties have been widely recognized (5-10). The surrounding stroma or tumor microenvironment is composed of an altered extracellular matrix, and a wide variety of cell types, including resident epithelial cells, fibroblasts, smooth muscle cells, immune and inflammatory cells, and lymphatic and vascular endothelial cells $(11,12)$. A population of surrounding fibroblasts known as cancer-associated fibroblasts (CAFs) play a critical role in tumor progression by stimulating survival and proliferation signaling pathways, through heterotypic signaling with cancer cells $(13,14)$.

Therefore, PDAC is formed by neoplastic cancer cells and stromal cells, and the development of the tumor requires reciprocal communication and interactions among all cell types, which cause alterations in each other by mechanisms that remain poorly understood (15). Moreover, during tumor progression, microenvironment progressively changes to a cancer-associated state that promotes tumor growth and metastasis. On the other hand, pancreatic cancer is a disease characterized by a highly oncogenic genetic profile (16-18). Consequently, tumors are heterogeneous tissues composed of different cells with distinct characteristics, and individual tumor cells of the same tumor have different tumorigenic potential $(11,19)$.

Biological information between cells can also be transferred through small extracellular vesicles (EV; exosomes, microparticles, oncosomes, etc.) released into interstitial spaces, which can be considered a snapshot of the cell's inner contents. They are being intensively studied as promising biomarkers for treatment selection and monitoring for several cancers (20-22).

In this study, to elucidate the role of tumor-stromal interactions in modulating pancreatic cancer progression, the effects on migration and invasion properties of different co-cultures conditions between human PDAC cell lines (Capan-1 and PL45) and fibroblasts (LC5), have been studied.

\section{Methods}

\section{Cell culture and chemical reagents}

PL-45 and Capan-1 human pancreatic cancer cell lines were obtained from LGC-ATCC (Barcelona, Spain), and LC5 embryonic fibroblasts cell line was kindly provided by Dr. G. Criado (Instituto de Investigación Hospital 12 de Octubre, Madrid). Differential genetic characteristics between tumor cell lines included in the study are shown in Supplementary Table 1. Cell lines were grown according to ATCC instructions, at $37^{\circ} \mathrm{C}$ in humidified atmosphere with $5 \% \mathrm{CO}_{2}$. IMDM and RPMI cell media, fetal bovine serum (FBS), penicillin, streptomycin and L-glutamine were obtained from Lonza Ltd. (Verviers, BE).

\section{Establishment of LC5 cell line constitutively expressing EGFP}


Lentivirus was generated by co-transfection of pRRL.SIN-EF1a-PGK-EGFP (encoding green fluorescent protein; \#12252) with pRSV-Rev (\#12253), pMD2.VSVg (\#12259), and pMDL.gag/pol.RRE (\#12251) plasmids in 293T cells. After 48h, viral supernatants were collected, filtered and frozen at -80 until use. LC5 cell line was infected for $24 \mathrm{~h}$ with $4 \mathrm{~mL}$ of virus-containing medium in presence of $10 \mu \mathrm{g} / \mathrm{mL}$ polybrene. Selection and isolation of fibroblasts-GFP ${ }^{+}$was performed with Sony Synergy Cell Sorter separation device (Sony Biotechnology Inc.), and based of GFP expression, and incorporation of Propidium lodide (PI) for the exclusion of dead cells. All plasmids required were from non-profit plasmid repository Addgene (http://www.addgene.org/).

\section{Co-cultures of fibroblasts and pancreas cancer cell lines}

For direct co-cultures between pancreatic tumor cell lines and fibroblasts, cells were cultured in a ratio 1:1, in RPMI medium supplemented with $10 \%$ FBS. Tumor pancreatic cells were seeded 72 hours before fibroblasts were added to the culture. The optimum time for these direct co-cultures was experimentally established in $72 \mathrm{~h}$, to obtain a final ratio of tumor cells:fibroblasts of 20:80, reflecting what is described in vivo. For functional analysis, cells were separated by cell sorting based of GFP expression and incorporation of PI.

Conditioned media (CM) were prepared from fibroblasts and pancreatic cancer cell lines. Cells were grown in $75 \mathrm{~cm}$ flasks until semiconfluent conditions, washed three times with phosphate-buffered saline (PBS), and then incubated in $1 \%$ of FBS medium for another $48 \mathrm{~h}$. Next, supernatants were collected, centrifuged at $2000 \mathrm{rpm}$ for $10 \mathrm{~min}$, sterile filtered $(0.45 \mu \mathrm{m}$ pore), and immediately used for assays of indirect co-cultures.

\section{Wound healing, migration, and invasion assays}

For wound-healing assay, cells were seeded on 6-well plates, and left to reach $80 \%$ confluence. A single wound per well was scratched with a sterile $200 \mu \mathrm{L}$ pipette tip within the monolayers and then washed with PBS to remove detached cells. CM from tumor cells was added to fibroblasts, and similarly CM from fibroblasts was added to tumor cells, and the rate of wound closure was observed in the following 24,48 , and 72 hours. Wound closure was monitored using a Nikon TE2000-S microscope.

Transwell migration assays were carried out using $8.0 \mu \mathrm{m}$ polycarbonate cell culture inserts (Transwell $\circledast$ Permeable Supports, Corning Inc., NY). Cells were serum-starved $24 \mathrm{~h}$ before the start of the experiments. Tumor cells were seeded at $1 \times 10 \mathrm{e} 5$ per well in $2 \mathrm{~mL}$ growth medium in the upper chamber (cell culture inserts) of 6-well plates. The same number of fibroblasts was seeded in the lower wells. Cells were allowed to migrate for 72 hours toward the underside of the membrane to the lower chamber. After that, cells attached to the upper side of the filter were removed by wiping with a cotton swab, and the migrated cells on the lower surface were fixed with formaldehyde $4 \%$ in PBS and permeabilized with absolute 
methanol in darkness. After several washes with PBS, cell nuclei were stained with 4'-6-diamino-2phenylindole (DAPI).

We used Ibidi culture-insert 2 well in $\mu$-dish $35 \mathrm{~mm}$ plates, with a defined $500 \mu \mathrm{m}$ cell free gap (Inycom Biotech, Zaragoza, ES) for migration assays. Tumor cells were seeded on one side of the insert and fibroblasts-GFP ${ }^{+}$on the opposite side. After $24 \mathrm{~h}$ for cells adhesion, the insert was removed, and migration was allowed. This system allows us to observe the migratory behavior of each cell type under the indirect influence of the other.

\section{Western blot analysis}

Whole cell lysates were prepared using MCL1 lysis buffer in the presence of protease and phosphatase cocktail inhibitor (Sigma-Aldrich, St Louis, MO) following the manufacter's protocol. Protein lysates were subjected to SDS-PAGE on $10 \%$ polyacrylamide gel and transferred onto PVDF membranes. Membranes were probed for the protein levels of E-cadherin, and a-tubulin as loading control, using specific primary antibodies (\#4065, Cell Signaling Tech. Inc., Danvers, MA, and \#sc-5286, Santa Cruz Biotechnology, Inc., Dallas, TX, respectively). Specific bands for each protein were visualized by WesternBright ${ }^{\mathrm{Tm}} \mathrm{kit}^{\mathrm{A}}$ (Advansta, San Jose, CA).

\section{Enzyme-linked Immunosorbent Assay (ELISA) and human cytokine array.}

Culture supernatant was collected from each growth condition, and keratinocyte growth factor (KGF-7) concentration was determined using Quantikine human KGF/FGF-7 Immunoassay (R\&D Systems Inc., Minneapolis, MN). ExoQuick-TC $₫$ ULTRA EV Kit and ExoELISA-ULTRA CD63 Kit (System Biosciences, Palo Alto, CA) were used for extracellular vesicles isolation and quantification, respectively. For the semiquantitative detection of 23 human protein in cell culture media, the human cytokine antibody array $\mathrm{C} 1$ (AAH-CYT-1; RayBiotech, Norcross, GA), was used (Supplementary Figure 2). All determinations were performed according to the manufacturer's instructions.

\section{Confocal immunofluorescence microscopy}

Direct and indirect co-cultures of fibroblasts-GFP ${ }^{+}$and tumor pancreatic cells were seeded on glass coverslips. Cells were washed twice with warm PBS, fixed in $4 \%$ paraformaldehyde and processed for immunofluorescence staining. First, cells were incubated overnight with mouse anti-a-Smooth Muscle Actin (a-SMA) antibody ( $\mathrm{n} 1584$, Dako, Carpinteria, CA) at $4^{\circ} \mathrm{C}$. After thorough washing with PBS, cells were incubated for $1 \mathrm{~h}$ with Alexa Fluor ${ }^{\circledR}$ 594-conjugated goat anti-mouse antibody and DAPI. Cells were mounted with Fluorsave ${ }^{\mathrm{m}}$ Reagent (Calbiochem, San Diego, CA) and examined in a Zeiss Axioplan 2 confocal microscope. 


\section{Transmission electron microscopy (TEM)}

Cells were fixed in $2.5 \%$ glutaraldehyde at $\mathrm{pH} 7.2$ for $24 \mathrm{~h}$, and later in $1 \% 0$ s04 in a $0.1 \mathrm{M}$ cacodylate buffer for $1 \mathrm{~h}$. Then, the samples were spinned to obtain pellets. The pellets were fixed in $1 \%$ uranyl acetate for $\mathbf{3 0}$ minutes, were then dehydrated in a series of graded ethanol steps, and finally embedded in epoxy resin. Thin sections were performed and stained with toluidine blue. Ultrathin sections were obtained from representative areas and were double stained with lead citrate and uranyl acetate and viewed under a JEOL JEM-1011 microscope.

\section{Statistical analysis}

Each experiment was performed in triplicate in at least three independent experiments. The analysis of differences was performed using Mann-Whitney $U$ test and variance analysis for repeated measurement data. Results were analyzed using software GraphPad Prism version 6.0 (GraphPad software, San Diego, CA) and SPSS v21.0 (SPSS Inc., Chicago, IL). The value $P<0.05$ was considered as significant.

\section{Results}

\section{Co-cultures of pancreatic tumor cells and fibroblasts-GFP ${ }^{+}$}

First, we established a fibroblast cell line constitutively expressing GFP protein (LC5-GFP+) in which the percentage of fibroblasts-GFP ${ }^{+}$was higher than $97 \%$ (Supplementary Figure 1).

Experimental design and images from direct co-cultures between PDAC and LC5-GFP ${ }^{+}$cells at 72h, are shown in Fig. 1. It is noteworthy that fibroblasts in the vicinity of the tumor cells surface established close contacts to these cells, being so arranged around the tumor cells to maximize the contact between cell to cell.

With the aim of evaluating the importance of the cell-cell adhesion and juxtacrine signaling versus paracrine signaling between cancer and stromal cells in the pathophysiology of pancreatic cancer, directand indirect co-cultures with $\mathrm{CMs}$, were used in the following assays.

\section{Co-culture modifies the expression of activation markers in tumor cells and fibroblasts}

To check activation of fibroblasts, LC5-GFP ${ }^{+}$cells were seeded in direct co-cultures with tumor cells, or indirect co-cultures with CM from Capan-1 or PL-45 cells. Representative pictures of a-SMA immunofluorescence staining are shown in Fig. 2a. LC5-GFP', Capan-1, and PL-45 cells do not express the a-SMA activation marker when they grow alone at monocultures (rows 1, 2, and 5). However, we can see that fibroblasts express high level of a-SMA both in direct- and indirect-culture with tumor cells (rows 
3, 4, 6, and 7). Quantification of LC5-GFP+ cells expressing a-SMA is shown in Fig. 2b. However, this fibroblast activation did not result in increased tumor cell proliferation, since no significant differences between indirect- and monoculture conditions were found in cell proliferation assays (Fig. 2c).

In addition, different FGF-7 expression levels between cells grown alone or in co-cultures were found. Thus, FGF-7 levels in co-culture supernatants were higher than in monoculture supernatants were (Fig. 3a, $P<0.0001)$. These results show that fibroblasts under the co-culture conditions here described, acquire the so-called myofibroblast or CAF phenotype.

Similarly, tumor cells were also affected by the presence of fibroblasts in co-culture. To check the induction of epithelial mesenchymal transition (EMT) process, we isolated tumor cells by sorting, and quantified the protein levels of E-cadherin by immunoblotting (Fig. 3b-c). E-cadherin is a cell adhesion molecule necessary for maintenance of intercellular contacts and cellular polarity in epithelial tissues, and a key feature of EMT. We can see that in both direct and indirect co-cultures, pancreatic tumor cells decrease their expression of E-cadherin, which would be attributed to an EMT process.

\section{Cytokines secretion changes under co-culture conditions}

It has been reported that cytokines and growth factors secreted by CAF or tumor cells are associated with tumor invasion and migration. Thus, the effects of direct- and indirect-cocultivation of fibroblasts and tumor cells on cytokine production were examined next. We collected the supernatants from monocultures, direct- and indirect co-cultures, and quantified the differences in protein expression of 23 specific human cytokines by a human cytokine antibody array (Supplementary Figure 2, Fig. $3 \mathrm{~d}-\mathrm{g}$ ). Relative levels of the cytokines differentially expressed between fibroblasts, Capan-1 and PL-45 monocultures are shown in Fig. 3d. Then, we compared the expression of these proteins in direct- and indirect-cocultures versus respective monocultures (Fig. $3 e$, versus fibroblasts monoculture; $3 f$, versus Capan-1 monoculture; $3 \mathrm{~g}$, versus PL-45 monoculture). We found that the GM-CSF growth factor, and cytokines GRO a/b/g, GRO a, IL-6, IL-8 and RANTES were markedly increased in LC5-cancer cells cocultures compared to LC5 monoculture. Curiously, GM-CSF, GRO a/b/g and GRO $a$, show a considerable decrease only when LC5 cells are grown with CM of PL-45. It is noteworthy the high expression of IL-6 in co-cultures of fibroblasts and tumor cells compared to tumor cells monocultures (Fig. 3e-g). However, we observed an increase in chemokine RANTES and growth factor GM-CSF levels in co-culture of fibroblasts with Capan-1 cells, but a decrease in co-culture of fibroblast and PL-45 cells. The cocultivation of fibroblasts and PL-45 cells was the one that showed the most differences in the pattern of cytokine secretion depending on the co-culture conditions, mainly in relation to IL-8, GRO $a$ and GRO a/b/g levels.

\section{Co-culture modifies motility of tumor cells and fibroblasts}

The migratory behavior of cells in direct- or indirect co-culture was examined by wound-healing and transwell migration assays. Fig. 4a-c shows representative images of Capan-1 cells grown in direct co- 
culture with fibroblasts-GFP'. Both cell types migrate into the wound, but curiously, fibroblasts migrate predominantly as single cells, whereas tumor cells migrated as a tightly packed sheet surrounded by fibroblasts.

To explore whether cell-cell contact is necessary for migration activity, we cultured Capan-1 or PL-45 tumor cells with fibroblasts CM for 3 days, and vice versa, LC5 with CM from each tumor cell line, and assayed their migration activity to wound-healing (Fig. 4e-f). Only PL-45 cells exposed to LC5 supernatant showed a weak migration increase at $48 \mathrm{~h}$ (Fig. 4e).

On the other hand, Capan-1- and PL-45-CM differed in their ability to affect the movement of LC5 cells, with Capan-1-CM being a more potent inducer (Fig. 4f). It is noteworthy that fibroblasts grown with Capan-1-CM travel to the center of the wound, invading the entire field. In contrast, fibroblasts cultured with PL-45-CM do not leave the edge of the wound, suggesting that wound healing occurs rather by cell proliferation than by fibroblasts migration.

Next, Capan-1 or PL-45 cells were cultured in the upper chamber of transwell migration system for 3 days. The number of invasive tumor cells increased significantly when fibroblasts were seeded in the lower chamber, compared to complete growth medium as control. This is substantial in Capan-1 cell line, but even more so in the case of PL-45 tumor cell line (Fig. 4d).

Last, we carry out migration assays in the lbidi culture-insert plates. Tumor cells were seeded on one side of the insert and LC5-GFP ${ }^{+}$on the other one. As a control, LC5-GFP ${ }^{+}$cells were seeded on both sides of the insert (first column, Fig. 4g). Fibroblasts in co-culture with PL-45 cells have a migration similar to that observed in the control (Fig. 4g, column 4 and 5). However, fibroblasts in co-culture with Capan-1 cells increase their migratory capacity, leaving the edge of the culture and moving towards the tumor cells (Fig. $4 \mathrm{~g}$, column 2 and 3 ). Moreover, in the vicinity of contact between both cell types, fibroblasts are arranged so as to increase the surface area of contact with tumor cells. These results are in agreement with those obtained previously in direct- and indirect co-cultures.

\section{Identification and quantification of extracellular vesicles (EV) in co-cultures}

Since there is ample evidence that EVs play a crucial role in intercellular communication, we decided to study the secretion of these vesicles in our co-culture assays. Visualization was realized by TEM (Fig. 5ae). Although TEM is not a quantitative technique, and we cannot differentiate between tumor cells and fibroblasts in co-cultures, we found a higher number of EVs per cell under co-culture versus monoculture conditions. EVs displayed typical spherical morphology and fell within the expected size range of exosomes (30-150 nm). We therefore isolated and quantify EVs from supernatants samples, and we could see that exosomes from co-cultures have a higher amount of protein than those from monoculture (Fig. 5f). 


\section{Discussion}

Pancreatic tumors are heterogeneous tissues composed of tumor and non-tumor cells, embedded within a dense desmoplastic stroma $(6,23)$. There are clear evidences that stroma is not just a static mechanical barrier but is thought to be an active participant involved in tumor initiation, progression, and metastasis (24). Thus, the study of pancreatic cancer cells behavior in monocultures does not faithfully reflect what is observed in vivo, given that all tumor components have a significant impact on the biological properties of malignancy. In this regard, in the work here exposed we use in vitro co-culture models, in an attempt to recapitulate some of the stromal-tumor connections, as a mean to assess the impact of this partnership on migration and metastasis initiation in PDAC.

Fibroblasts are the most common component of tumor stroma, and CAFs provide a favorable microenvironment with tumor-promoting properties, stimulating the progression of primary tumors via paracrine signaling (25), and serving as a niche to support the metastatic colonization (26-28). Thus, the association between cancer cells and CAFs leads to alteration of the biological properties of both types of cells through bi-directional tumor stroma crosstalk.

In our co-culture model, KGF/FGF-7 secretion is upregulated and it has been postulated than CAFs could increase the viability and migration of tumor cells by a paracrine manner through this growth factor (29). In addition, a-SMA is the most widely used marker to asses CAFs activation status and is associated with worse clinical outcomes $(30,31)$.

Cell migration is an important factor in the spread of pancreatic cancer and is a key step in the series of events that lead to metastasis. In the wound-healing assays of direct co-culture, we can see that groups of cells move into the wound. In these clusters, fibroblasts are arranged surrounding tumor cells. Besides this curious migration formation, it is also noteworthy the amoeboid characteristics of tumor cells movement. Amoeboid migration facilitates cell position change without cell remodeling but move by low adhesion force and high cell deformability. Instead, collective migration takes place when cells retain cellcell adhesions and coordination among them. These migration modes are interconvertible, and tumor cells use both mechanisms in an adaptive way, exhibiting its great plasticity (32). In our work, this peculiar way of migrating set, tumor cells and surrounding fibroblasts in the middle of wound, could indicate that both types of cells actively cooperate to migrate to other organs, as a single pack of invasion. Previous reports have shown that single cancer cells may proliferate via interactions with stromal cells derived from other organs when they form metastatic tumors (33). However, these mechanisms are not mutually exclusive, and fibroblasts could come from either the primary tumor or metastatic sites.

Using traditional migration assay setup, we show that only Capan-1 cells were able to induce an important CAFs migration as individually moving cells. Similarly, Ishii et al., have reported that lung fibroblasts co-cultured with human cancer cell line Capan-1 showed significantly higher migration activity than fibroblasts alone (34). In addition, Karagiannis et al. showed in co-cultures of colon cancer cell lines and fibroblasts that the CAFs migratory behavior was context-dependent, varying from totally individual 
to collective configuration (35). Therefore, fibroblasts would have dissimilar migratory responses upon stimulation with different pancreatic cancer cells, such as Capan-1 or PL-45.

Activated fibroblasts can promote invasive growth by cell-cell contacts or by paracrine diffusible signals, which also can induce EMT of tumor cells $(36,37)$. On the other hand, we have shown an important decrease in the expression of the epithelial marker E-cadherin in tumor cells from our co-culture model, suggesting that EMT occurs in this system and would facilitate the initiation of metastasis (4). However, variable expression of E-cadherin in human pancreatic cancer samples and commercial cell lines has been confirmed in several studies, supporting the hypothesis that PDAC cells exhibit a hybrid EMT-related phenotype (38).

In the crosstalk between fibroblasts and cancer cells, two closely pathway have been described: one pathway where stroma cells affect cancer cells response and other where cancer cells trigger a response in the stroma cells $(37,39)$. Both responses could be mediated by different secretion of cytokines from the cells involved. Furthermore, we found that the level of IL-6 mainly, in addition to GM-CSF and RANTES, changed significantly in co-cultures conditions with respect to monocultures, and this could affect the invasive and migratory capacities of cells. Increased IL-6 level has previously been related to protumorigenic function of CAFs, promoting contractibility and extracellular matrix remodeling $(40,41)$. Curiously, we have not found change in TGF- $\beta$ level between the different co-culture conditions. Even more when IL- 6 is also a common cytokine that enhances TGF- $\beta$ signaling, resulting in EMT and stimulates tumor progression. However, TGF- $\beta$ signaling has multifaceted and cell type specific in PDAC (42). Thus, into the heterogeneous cancer-associated fibroblasts population, there may be a subset of inflammatory CAFs expressing pro-inflammatory cytokines, which could be majority in our co-culture models.

Exosomes have recently been described as communication tools between cells, and they could mediate interactions between tumor cells and the microenvironment $(22,43)$. They are secreted from almost all cell types although tumor cells secrete more EVs compared to normal cells, playing an essential role in inducing metastasis and remodeling the primary microenvironment. Thus, it has been shown that EVs from fibroblasts promote invasive and chemoresistant behavior in cancer cells $(44,45)$, whereas EVs from tumor cells can reprogram fibroblasts into CAFs. In addition, it has been described that an increased amount of protein per exosomes predict disease progression $(46,47)$. Our results are in agreement with previously published data since they indicate that cells in co-cultures produce a greater number of exosomes, and these possess a higher amount of protein that cells in monoculture.

A growing body of literature dealing on the tumor-stroma interactions, point to the future approach for cancer treatments based against both malignant and microenvironmental cells $(24,48,49)$. Since pancreatic cancer cells are surrounded by a thick and poorly perfused stroma, which halts penetration of otherwise effective treatments, it would be reasonable to think that incorporating a stromal depleting agent into therapeutic treatments, or disrupting the crosstalk between stroma and cancer cells, would 
sensitize chemoresistant tumors and improve the outcome of patients (50-52). Similarly, exosomes could be used in antitumor therapies by exploiting their characteristic properties (53).

Finally, an important limitation of our study is that it focuses on cancer-associated fibroblasts as the leading stromal element interacting with pancreatic tumor cells. However, many other cell types in the stroma are known to influence tumor cells behavior, such as immune and inflammatory cells, adipocytes, and endothelial cells. Two different PDAC cell lines were used in our co-culture model, and we can not generalize the results to the entire spectrum of pancreatic cancer. In this study, we have achieved some relevant results, but also accepting the incomplete approximation of the in vitro set-ups, as they are not always able to simulate the high tissue complexity found in vivo.

\section{Conclusions}

Collectively, we have shown a co-culture model of tumor cells and fibroblasts, which recapitulates some features occurring in human PDAC, and might be used for a better understanding of tumor-stromal interactions and identify new targets for pharmacological intervention. We show different migratory properties in tumor and stromal cells, and a different mechanism for tumor spread through the joint migration of tumor cells and fibroblasts. Taken together, our results suggest that direct contact between fibroblasts and Capan-1 cells is involved in the progression of tumor, in addition to the paracrine promoting effect of growth factor or chemokines such as GM-CSF or IL-6. However, CAFs could lead PL45 migration mainly though paracrine mechanisms, besides juxtacrine ones. Although cancer-stroma interaction may be a promising target for new therapeutic approaches, these should focus on each tumor histotype, because of the variability of tumor cells and the role played by CAFs in each tumor.

\section{Abbreviations}

a-SMA: a-Smooth Muscle Actin; CAFs: Cancer-associated fibroblasts; CM: Conditioned media; EMT: Epithelial-to-Mesenchymal Transition; EV: extracellular vesicles; GFP: Green fluorescent protein; KGF/FGF7: Keratinocyte growth factor/Fibroblast growth factor 7; LC5-GFP+: LC5 fibroblasts expressing Green fluorescent protein; PDAC: Pancreatic ductal adenocarcinoma; TEM: Transmission electron microscopy; TGF- $\beta$ : Transforming growth factor- $\beta$.

\section{Declarations}

\section{Ethics approval and consent to participate}

Not applicable.

\section{Consent for publication}

Not applicable. 


\section{Availability of data and materials}

The datasets used and/or analyzed during the current study are available from the corresponding author on reasonable request.

\section{Competing interests}

The authors declare that they have no competing interests.

\section{Funding}

This study was supported by Fundación Médica Mutua Madrileña (06/0137). The sponsor had no role in study design, data collection, data analysis, data interpretation, writing of the paper and in the decision to submit the article for publication.

\section{Authors' contributions}

CG, JA, JAL-M, and MTA-O conceived and designed the study. EP-G, CVD-G, AA-L, VP-M, IG-C, SA-P, MA-R, and $C P$ conducted experiments. EP-G, LP-A, and MTA-O analyzed and interpreted data. All authors read and approved the final manuscript.

\section{Acknowledgements}

The authors thanks Fernando Bartolomé (Neurodegenerative Diseases, i+12) for technical assistance.

\section{References}

1. Siegel RL, Miller KD, Jemal A. Cancer Statistics. 2017. CA: a cancer journal for clinicians. 2017;67:730.

2. Hidalgo M, Alvarez R, Gallego J, Guillen-Ponce C, Laquente B, Macarulla T, et al. Consensus guidelines for diagnosis, treatment and follow-up of patients with pancreatic cancer in Spain. Clin Transl Oncol. 2017;19:667-81.

3. Taieb J, Pointet AL, Van Laethem JL, Laquente B, Pernot S, Lordick F, et al. What treatment in 2017 for inoperable pancreatic cancers? Ann Oncol. 2017;28:1473-83.

4. Palumbo A Jr, Costa Nde D, Bonamino O, Pinto MH, Nasciutti LF. LE. Genetic instability in the tumor microenvironment: a new look at an old neighbor. Mol Cancer. 2015;14:145.

5. Neesse A, Michl P, Frese KK, Feig C, Cook N, Jacobetz MA, et al. Stromal biology and therapy in pancreatic cancer. Gut. 2011;60:861-8. 
6. Erkan M, Hausmann S, Michalski CW, Fingerle AA, Dobritz M, Kleeff $\mathrm{J}$, et al. The role of stroma in pancreatic cancer: diagnostic and therapeutic implications. Nature reviews Gastroenterol Hepatol. 2012;9:454-67.

7. Apte MV, Wilson JS, Lugea A, Pandol SJ. A starring role for stellate cells in the pancreatic cancer microenvironment. Gastroenterology. 2013;144:1210-9.

8. Hu H, Jiao F, Han T, Wang LW. Functional significance of macrophages in pancreatic cancer biology. Tumour Biol. 2015;36:9119-26.

9. Pickard A, Cichon AC, Barry A, Kieran D, Patel D, Hamilton P, et al. Inactivation of Rb in stromal fibroblasts promotes epithelial cell invasion. EMBO J. 2012;31:3092-103.

10. Elyada E, Bolisetty M, Laise P, Flynn WF, Courtois ET, Burkhart RA, et al. Cross-Species Single-Cell Analysis of Pancreatic Ductal Adenocarcinoma Reveals Antigen-Presenting Cancer-Associated Fibroblasts. Cancer Discov. 2019;9:1102-23.

11. Ligorio M, Sil S, Malagon-Lopez J, Nieman LT, Misale S, Di Pilato M, et al. Stromal Microenvironment Shapes the Intratumoral Architecture of Pancreatic Cancer. Cell. 2019;178:160-75 e27.

12. Froeling FE, Marshall JF, Kocher HM. Pancreatic cancer organotypic cultures. J Biotechnol. 2010;148:16-23.

13. Hwang RF, Moore T, Arumugam T, Ramachandran V, Amos KD, Rivera A, et al. Cancer-associated stromal fibroblasts promote pancreatic tumor progression. Cancer Res. 2008;68:918-26.

14. Fiori ME, Di Franco S, Villanova L, Bianca P, Stassi G, De Maria R. Cancer-associated fibroblasts as abettors of tumor progression at the crossroads of EMT and therapy resistance. Mol Cancer. 2019;18:70.

15. Kiaris $H$, Trimis $G$, Papavassiliou AG. Regulation of tumor-stromal fibroblast interactions: implications in anticancer therapy. Curr Med Chem. 2008;15:3062-7.

16. Deer EL, Gonzalez-Hernandez J, Coursen JD, Shea JE, Ngatia J, Scaife CL, et al. Phenotype and genotype of pancreatic cancer cell lines. Pancreas. 2010;39:425-35.

17. Xia X, Wu W, Huang C, Cen G, Jiang T, Cao J, et al. SMAD4 and its role in pancreatic cancer. Tumour Biol. 2015;36:111-9.

18. Chen YW, Hsiao PJ, Weng CC, Kuo KK, Kuo TL, Wu DC, et al. SMAD4 loss triggers the phenotypic changes of pancreatic ductal adenocarcinoma cells. BMC Cancer. 2014;14:181.

19. Maier $\mathrm{HJ}$, Wirth $\mathrm{T}$, Beug $\mathrm{H}$. Epithelial-mesenchymal transition in pancreatic carcinoma. Cancers. 2010;2:2058-83.

20. Han L, Lam EW, Sun Y. Extracellular vesicles in the tumor microenvironment: old stories, but new tales. Mol Cancer. 2019;18:59.

21. Willms E, Johansson HJ, Mager I, Lee Y, Blomberg KE, Sadik M, et al. Cells release subpopulations of exosomes with distinct molecular and biological properties. Sci Rep. 2016;6:22519.

22. Qiu J, Yang G, Feng M, Zheng S, Cao Z, You L, et al. Extracellular vesicles as mediators of the progression and chemoresistance of pancreatic cancer and their potential clinical applications. Mol 
Cancer. 2018;17:2.

23. Stromnes IM, DelGiorno KE, Greenberg PD, Hingorani SR. Stromal reengineering to treat pancreas cancer. Carcinogenesis. 2014;35:1451-60.

24. Klemm F, Joyce JA. Microenvironmental regulation of therapeutic response in cancer. Trends Cell Biol. 2015;25:198-213.

25. Kalluri R. The biology and function of fibroblasts in cancer. Nat Rev Cancer. 2016;16:582-98.

26. Han Y, Zhang Y, Jia T, Sun Y. Molecular mechanism underlying the tumor-promoting functions of carcinoma-associated fibroblasts. Tumour Biol. 2015;36:1385-94.

27. Ferrari N, Ranftl R, Chicherova I, Slaven ND, Moeendarbary E, Farrugia AJ, et al. Dickkopf-3 links HSF1 and YAP/TAZ signalling to control aggressive behaviours in cancer-associated fibroblasts. Nat Commun. 2019;10:130.

28. Vennin C, Melenec $P$, Rouet R, Nobis M, Cazet AS, Murphy KJ, et al. CAF hierarchy driven by pancreatic cancer cell p53-status creates a pro-metastatic and chemoresistant environment via perlecan. Nat Commun. 2019;10:3637.

29. Lin J, Liu C, Ge L, Gao Q, He X, Liu Y, et al. Carcinoma-associated fibroblasts promotes the proliferation of a lingual carcinoma cell line by secreting keratinocyte growth factor. Tumour Biol. 2011;32:597-602.

30. Froeling FE, Mirza TA, Feakins RM, Seedhar A, Elia G, Hart IR, et al. Organotypic culture model of pancreatic cancer demonstrates that stromal cells modulate E-cadherin, beta-catenin, and Ezrin expression in tumor cells. T Am J Pathol. 2009;175:636-48.

31. Owusu BY, Vaid M, Kaler P, Klampfer L. Prognostic and Predictive Significance of Stromal Fibroblasts and Macrophages in Colon Cancer. Biomark Cancer. 2015;7(Suppl 1):29-37.

32. Odenthal J, Takes R, Friedl P. Plasticity of tumor cell invasion: governance by growth factors and cytokines. Carcinogenesis. 2016;37:1117-28.

33. Fujita H, Ohuchida K, Mizumoto K, Egami T, Miyoshi $K$, Moriyama T, et al. Tumor-stromal interactions with direct cell contacts enhance proliferation of human pancreatic carcinoma cells. Cancer Sci. 2009;100:2309-17.

34. Ishii G, Hashimoto H, Asada K, Ito T, Hoshino A, Fujii S, et al. Fibroblasts associated with cancer cells keep enhanced migration activity after separation from cancer cells: a novel character of tumor educated fibroblasts. Int J Oncol. 2010;37:317-25.

35. Karagiannis GS, Schaeffer DF, Cho CK, Musrap N, Saraon P, Batruch I, et al. Collective migration of cancer-associated fibroblasts is enhanced by overexpression of tight junction-associated proteins claudin-11 and occludin. Molecular Oncol. 2014;8:178-95.

36. Cirri P, Chiarugi P. Cancer associated fibroblasts: the dark side of the coin. Am J Cancer Res. 2011;1:482-97.

37. Fujiwara A, Funaki S, Fukui E, Kimura K, Kanou T, Ose N, et al. Effects of pirfenidone targeting the tumor microenvironment and tumor-stroma interaction as a novel treatment for non-small cell lung 
cancer. Sci Rep. 2020;10:10900.

38. Sommariva M, Gagliano N. E-Cadherin in Pancreatic Ductal Adenocarcinoma: A Multifaceted Actor during EMT. Cells. 2020;9:1040.

39. Astekar M, Metgud R, Sharma A, Soni A. Hidden keys in stroma: Unlocking the tumor progression. J Oral Maxillofac Pathol. 2013;17:82-8.

40. Yoshida GJ. Regulation of heterogeneous cancer-associated fibroblasts: the molecular pathology of activated signaling pathways. J Exp Clin Cancer Res. 2020;39:112.

41. Öhlund D, Handly-Santana A, Biffi G, Elyada E, Almeida AS, Ponz-Sarvise M, et al. Distinct populations of inflammatory fibroblasts and myofibroblasts in pancreatic cancer. $\mathrm{J}$ Exp Med. 2017;214:579-96.

42. Huang $\mathrm{H}$, Zhang Y, Gallegos V, Sorrelle N, Zaid MM, Toombs J, et al. Targeting TGF $\beta R 2$-mutant tumors exposes vulnerabilities to stromal TGF $\beta$ blockade in pancreatic cancer. EMBO Mol Med. 2019;11:e10515.

43. Jin $\mathrm{H}, \mathrm{Wu} \mathrm{Y}, \operatorname{Tan} \mathrm{X}$. The role of pancreatic cancer-derived exosomes in cancer progress and their potential application as biomarkers. Clin TransI Oncol. 2017;19:921-30.

44. Hu JL, Wang W, Lan XL, Zeng ZC, Liang YS, Yan YR, et al. CAFs secreted exosomes promote metastasis and chemotherapy resistance by enhancing cell stemness and epithelial-mesenchymal transition in colorectal cancer. Mol Cancer. 2019;18:91.

45. Ariston Gabriel AN, Wang F, Jiao Q, Yvette U, Yang X, Al-Ameri SA, et al. The involvement of exosomes in the diagnosis and treatment of pancreatic cancer. Mol Cancer. 2020;19:132.

46. Peinado H, Aleckovic M, Lavotshkin S, Matei I, Costa-Silva B, Moreno-Bueno G, et al. Melanoma exosomes educate bone marrow progenitor cells toward a pro-metastatic phenotype through MET. Nat Med. 2012;18:883-91.

47. Zhang C, Ji Q, Yang Y, Li Q, Wang Z. Exosome: Function and Role in Cancer Metastasis and Drug Resistance. Technol Cancer Res Treat. 2018;17:1533033818763450.

48. Celli JP. Stromal interactions as regulators of tumor growth and therapeutic response: A potential target for photodynamic therapy? Isr J Chem. 2012;52:757-66.

49. Wang Q, Qu C, Xie F, Chen L, Liu L, Liang X, et al. Curcumin suppresses epithelial-to-mesenchymal transition and metastasis of pancreatic cancer cells by inhibiting cancer-associated fibroblasts. Am J Cancer Res. 2017;7:125-33.

50. Schober M, Jesenofsky R, Faissner R, Weidenauer C, Hagmann W, Michl P, et al. Desmoplasia and chemoresistance in pancreatic cancer. Cancers. 2014;6:2137-54.

51. Von Hoff DD, Ervin T, Arena FP, Chiorean EG, Infante J, Moore M, et al. Increased survival in pancreatic cancer with nab-paclitaxel plus gemcitabine. N Engl J Med. 2013;369:1691-703.

52. Kindler HL, loka T, Richel DJ, Bennouna J, Letourneau R, Okusaka T, et al. Axitinib plus gemcitabine versus placebo plus gemcitabine in patients with advanced pancreatic adenocarcinoma: a doubleblind randomised phase 3 study. Lancet Oncol. 2011;12:256-62. 
53. Steinbichler TB, Dudas J, Skvortsov S, Ganswindt U, Riechelmann H. Skvortsova, II. Therapy resistance mediated by exosomes. Mol Cancer. 2019;18:58.

\section{Figures}

Experimental design: direct co-culture
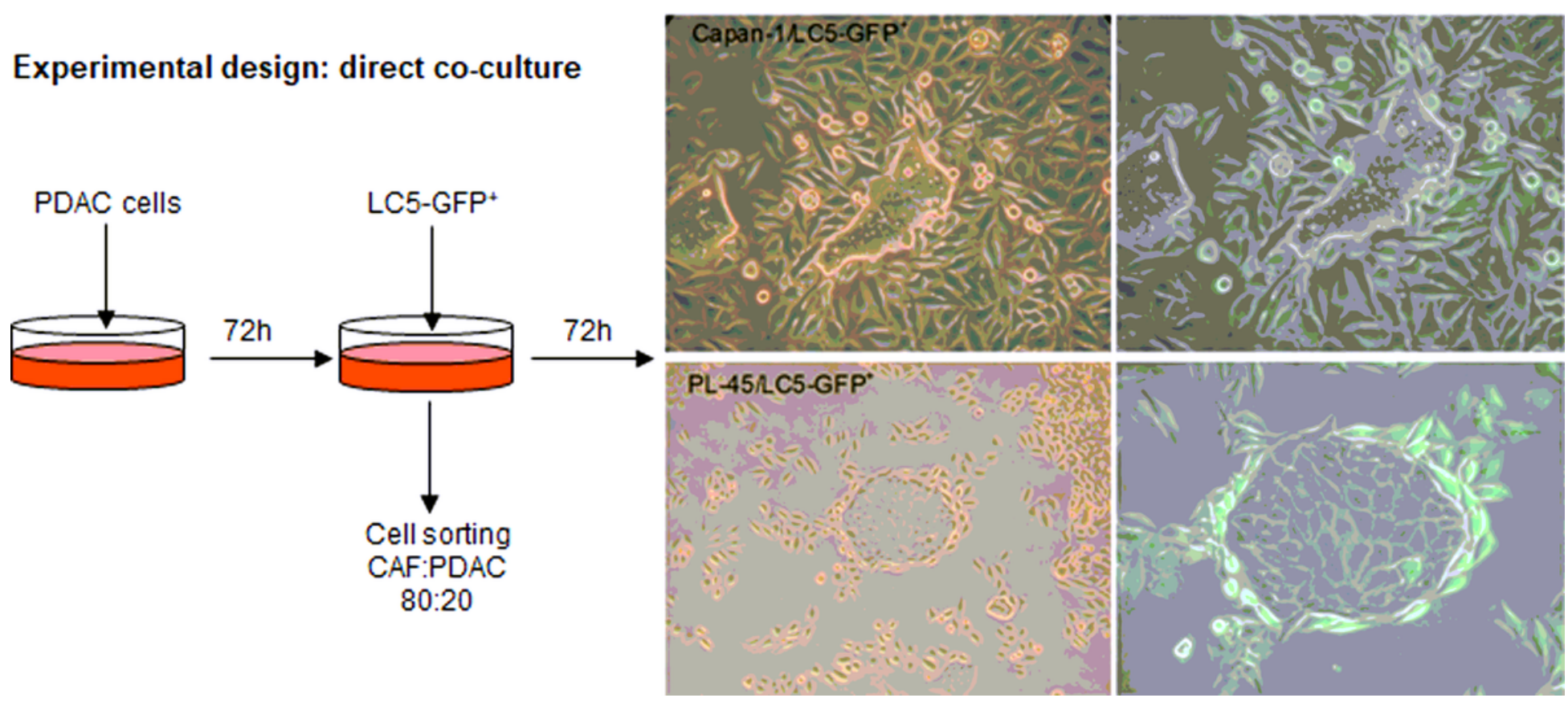

Figure 1

Strategy scheme of direct co-cultures: representative images at $72 \mathrm{~h}$ of pancreatic tumor cells (Capan-1 or PL-45) and fibroblasts LC5-GFP+. 

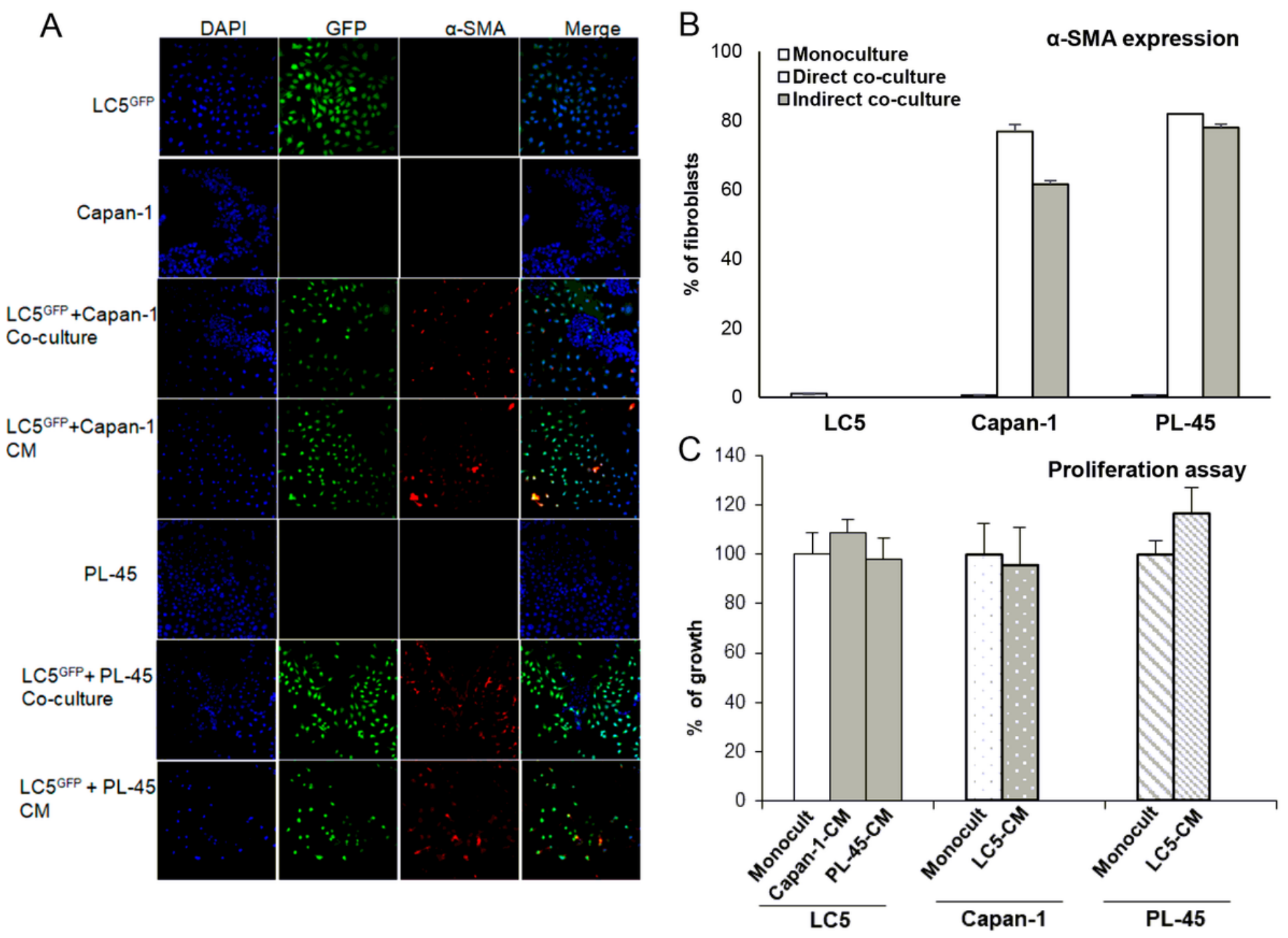

Figure 2

-culture modifies the expression of activation markers in tumor cells and fibroblasts, without affecting proliferation. a. Activated fibroblasts LC5-GFP+ express a-SMA in direct and indirect co-cultures with pancreatic tumor cells lines. Rows 1, 2, and 5: indicated cells in monoculture; rows 3 and 4: LC5GFP in direct- or indirect-coculture respectively, with Capan-1 pancreatic tumor cell line; rows 6 and 7: LC5GFP in direct- and indirect-co-culture with PL-45 pancreatic tumor cell line. b. Representative bar graph showing percentage of fibroblasts expressing a-SMA, in direct- or indirect-coculture respect to monoculture. Data obtained from three independent experiments and reported as mean \pm SD. c. Proliferation assays of cells growing in conditioned medium from fibroblasts or tumor cells, as indicated, respect to cells growing as monocultures. Data are presented as the mean \pm SD of three independent experiments. LC5GFP: LC5 fibroblasts expressing GFP protein. CM: Conditioned medium. 

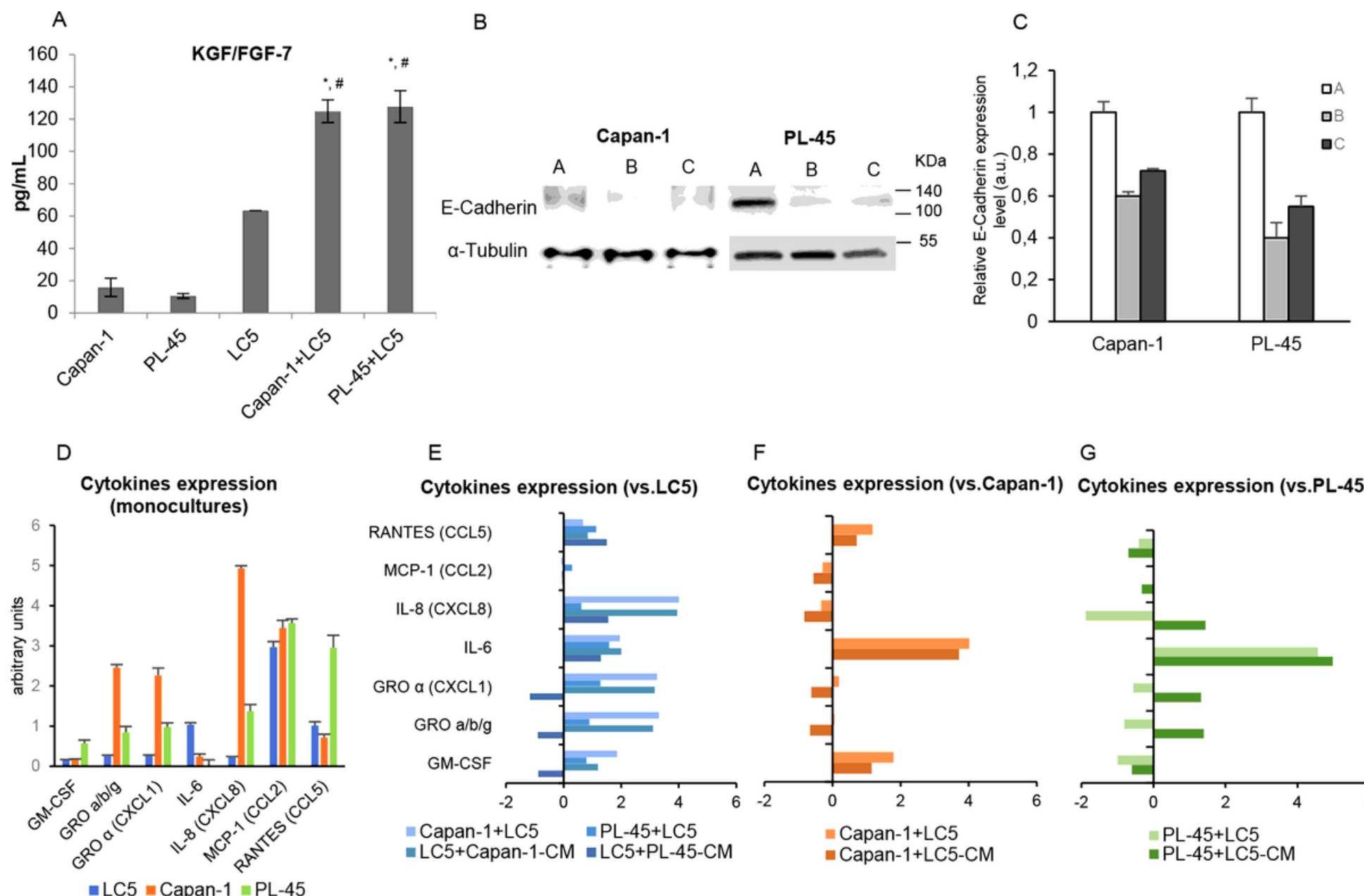

E

Cytokines expression (vs.LC5)

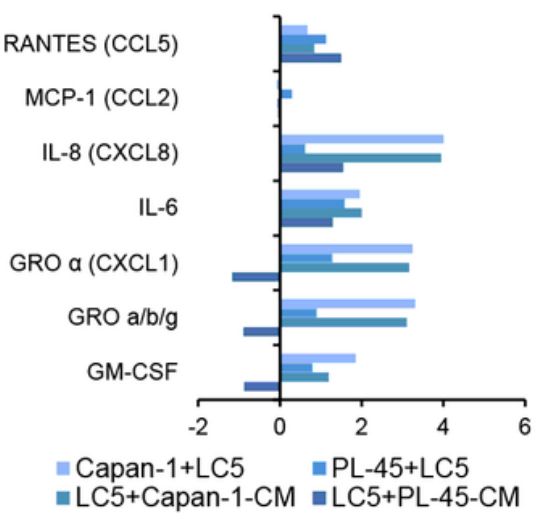

Cytokines expression (vs.Capan-1)

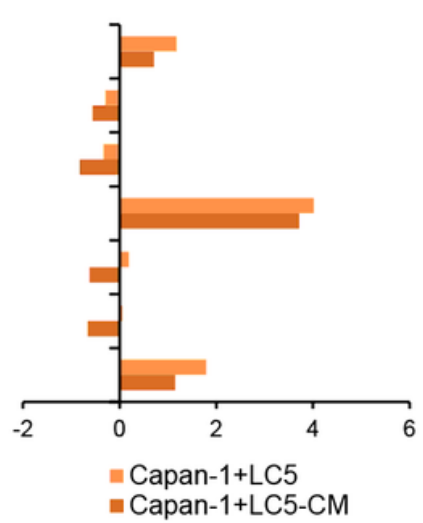

G

Cytokines expression (vs.PL-45)

\section{Figure 3}

Changes in protein expression and cytokines secretion in co-culture models. a. KGF/FGF-7 concentration from supernatants of monoculture and fibroblasts-pancreatic tumor cells co-cultures. FGF-7 concentration in co-cultures was significantly higher than that in monocultures (* respect to LC5, \# respect to the corresponding tumor cell line; $P<0.0001$ ). Data represent mean $\pm S D$ of three independent assays. b. Expression of EMT marker E-cadherin in pancreatic tumor cells by immunoblotting. Loading control a-Tubulin. c. Representative quantification of E-cadherin expression from western blot. A: cells grown alone at monoculture. B: cells grown at co-cultures with fibroblasts (protein extracts obtained after sorting), and C: cells grown with CM from fibroblast. d. Quantification of secretome dot blots from LC5, Capan-1 and PL-45 cells growing as monocultures, according to AAH-CYT-1 human cytokine antibody array. Representation of the molecules most significant expressed. eg. Relative differences in cytokines expression levels compared to LC5 monoculture (e), compared to Capan-1 monoculture (f), and compared to PL-45 monoculture (g). X-axis values shown are log2 of the normalized levels. 

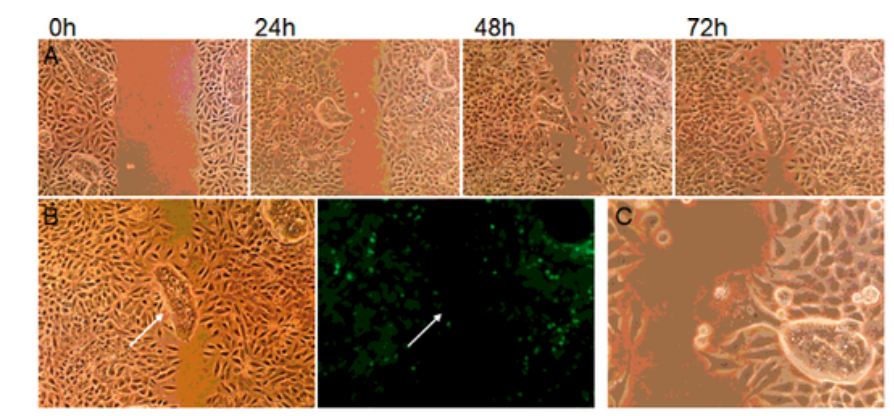

$\mathrm{F}$

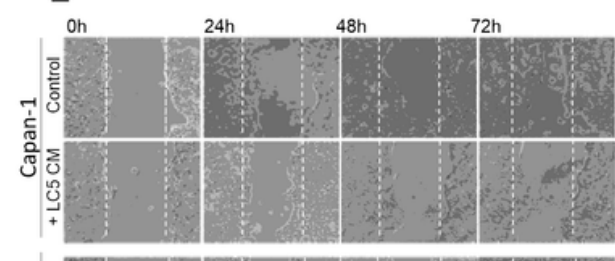

๕.

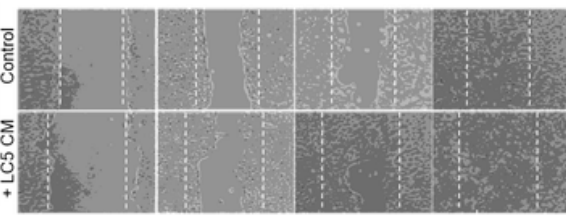

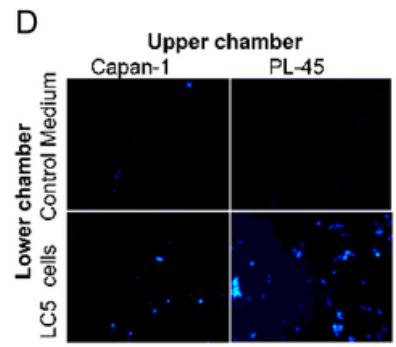

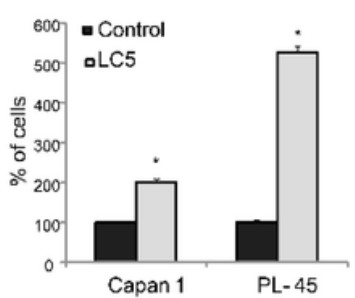

G
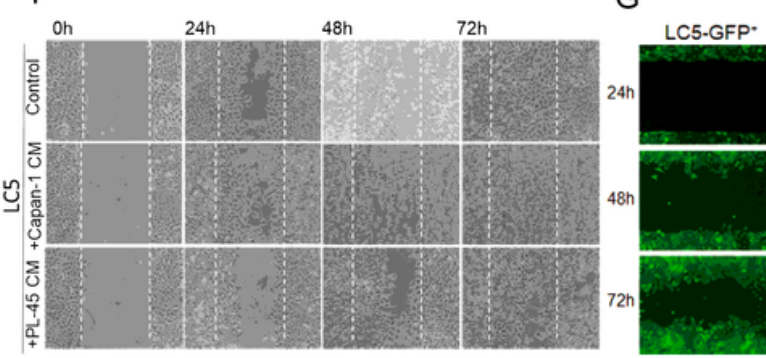

PL-45/LC5-GFP*

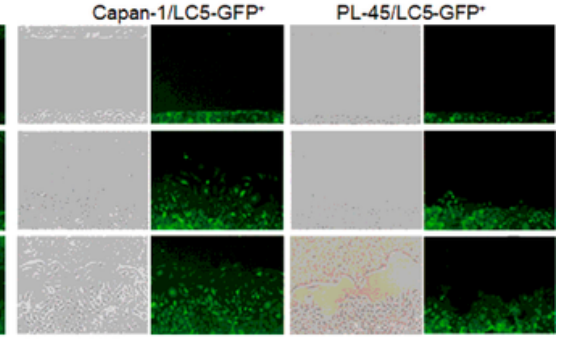

Figure 4

Migration and invasion assays in co-cultures conditions. a. Wound-healing assay in direct co-cultures of LC5-GFP+ and Capan-1 tumor cell line. Photographs taken after 24, 48, and 72 hours of co-culture. b. Images of cells migration in phase contrast (left) and fluorescence microscopes (right). c. Detail of tumor cells invading the wound. Enlarged figure depict tumor cells that are polarized with cytoplasmic protrusions facing the wound, in an amoeboid movement. $d$. Transwell migration assay. Representative photographs of Capan-1 and PL-45 cells that have invaded into the 8- $\mu \mathrm{m}$ pore membrane filter, and histograms of migrated tumor cells compared to control (100\%). Migrated cells were quantitated by counting cells in 10 randomly fields in each sample. Data are presented as mean \pm SD from three independent experiments $\left({ }^{*} P<0.0001\right)$.e. Wound scratch assay: treatment of pancreatic tumor cell lines with conditioned medium (CM) from fibroblasts. Control: tumor cells in growth medium. f. Treatment of LC5 fibroblasts with conditioned medium (CM) from Capan-1 or PL-45 pancreatic tumor cell lines.

Control: tumor cells in growth medium. g. Wound-healing assay in 2 well Ibidi culture-insert. First column: images in fluorescence microscope of cell migration at 24, 48 and 72h of LC5-GFP+ cells seeded on both sides of the insert. Columns 2 and 3: images of cell migration in phase contrast (column 2) and fluorescence microscopes (column 3 ) of Capan-1 tumor cells seeded in the upper side of the image and LC5-GFP+ seeded in the lower side. Columns 4 and 5: images of migration with PL-45 tumor cells in the upper side and LC5-GFP+ in the lower side. 

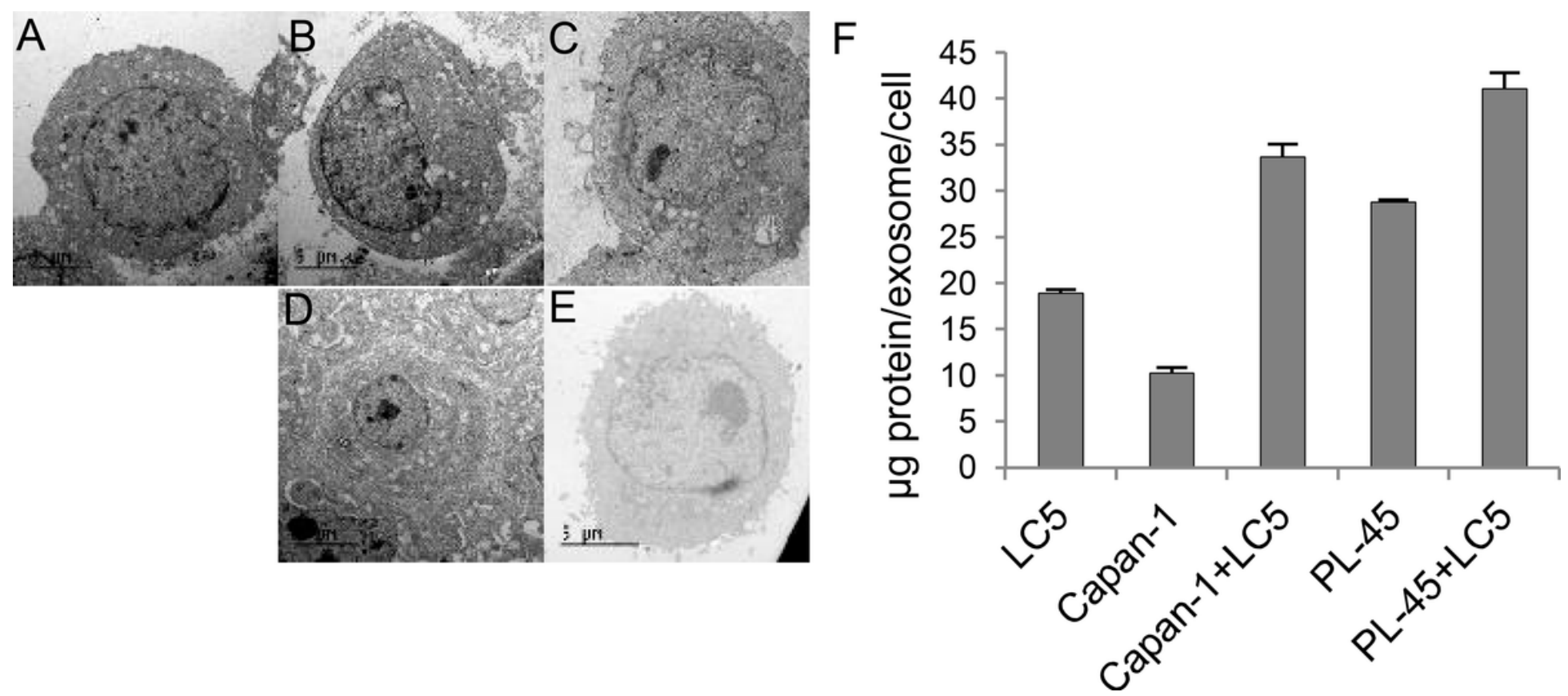

Figure 5

Representative images of cells growing during $72 \mathrm{~h}$, by transmission electron microscopy (TEM). a. LC5 cells growing at monoculture. b. Capan-1 cells in monoculture. c. PL-45 cells in monoculture. d. Capan-1 and LC5 cells in co-culture. e. PL-45 and LC5 cells in co-culture. f. Quantification of protein by exosome in monocultures and co-cultures.

\section{Supplementary Files}

This is a list of supplementary files associated with this preprint. Click to download.

- SupplementaryFigure1.docx

- SupplementaryFigure2.docx

- SupplementaryTable1.docx 